\title{
Astronomical education in Armenia
}

\author{
Hayk A. Harutyunian ${ }^{1}$ \\ ${ }^{1}$ Byurakan Astrophysical Observatory, Armenia \\ email: hhayk@bao.sci.am
}

\begin{abstract}
Astronomy pupils in Armenia get their first ideas on astronomy at elementary schools. Astronomy as a distinct subject is taught at all secondary schools in the country. Teaching is conducted according to a unified program elaborated jointly by professional astronomers and astronomy teachers. Unfortunately only one hour per week is allotted for teaching astronomy, which obviously is not enough workload to hire specialized astronomy teachers at every school, and at many schools this subject is tutored by non-specialists. Many schools partly compensate this lack of teachers by organizing visits to the Byurakan Astrophysical Observatory (BAO) for pupils, where they also attend short lectures on astronomy. In some schools optional training in astronomy is organized by amateurs, for the purpose of a deeper understanding in astronomy.

During recent years annual competitions for revealing gifted pupils in astronomy have been organized. These competitions have three rounds, namely, in schools, in districts and the final round is, as a rule, held at BAO. The national winners successfully participate in and win prestigious prizes at international astronomical Olympiads as well.

At Yerevan State University (YSU) there is a department for astrophysics, which was set up in 1946 and is operating to date. This department trains specialists for a career in astrophysics. Only one or two students graduate from this department yearly at present, while in the 1980s a dozen specialists were trained every year. BAO serves as the scientific base for the students of YSU as well, and a number of staff members from BAO conduct special courses for YSU students. YSU provides a Master's degree in astrophysics, and BAO is granting a Doctor's (PhD) degree since the 1970s.
\end{abstract}

Keywords. Astronomy education in Armenia, Byurakan Astrophysical Observatory

\section{Introduction and historical background}

Astronomical knowledge in Armenia has a rather long history. The first astronomical petroglyphs found in Armenia date to the V-IV millennia B.C. The ruins of the Metsamor ancient observatory are dated to the III-II millennia B.C. Written documents are rare, though one can find some astronomical ideas and knowledge in the "History of Armenia" by Movses Khorenatsy of the fifth century A.D. More systematically, astronomical ideas in Armenian had been given for the first time by Anania Shirakatsy of the seventh century. His manuscripts on astronomy, geography and mathematics served as textbooks for school pupils during centuries and one can study these books in the Matenadaran - the Institute of Ancient Manuscripts in Yerevan, the capital of Armenia, where many manuscripts of famous ancient Greeks are preserved as well.

\section{Astronomy education in Armenian high schools}

Astronomy as a separate subject was included into the secondary school program after 1920 (to the best of our knowledge) when the Soviet Armenian Republic was formed. All the Soviet republics have been implementing then the same educational programme approved by the Soviet Ministry of Education. During the second half of 20th century 
the astronomy textbook by Prof. Vorontsov-Velyaminov was the only mandatory course for all the pupils. In the 1970s an attempt was made to prepare another textbook in Armenia, taking into account the existence of rather high level professional astronomy in the country, and numerous good specialists available were ready to write a new textbook. Unfortunately this textbook has been in use for a few years only and the old one replaced it again very soon.

This situation changed dramatically after the Soviet Union collapsed, and every Soviet republic as a new independent country became free to carry out and implement its own programme, and any textbook could be adopted by the corresponding Ministry. Of course, such a change was welcomed with pleasure, not only by the astronomical community, and it is worth noting that with a few advantages gained as a result of independence, these countries including Armenia lost already or are losing some of the previous ones.

Turning to the present situation, one should mention that the first ideas in astronomy that pupils in Armenia get at elementary schools are owing to special hours prescribed for the introduction of elementary knowledge on the structure of the surrounding world. During about 10 hours, they learn about the geometric shape of the Earth, its rotation around its own axis and around the Sun, receive some information on the solar system structure and on other planets. The programme is prepared for 10-11 year old children to provide them with the most general information on the subject of astronomy.

At present astronomy as a distinct mandatory subject is taught at all secondary schools in the country. Teaching is conducted according to the unified programme elaborated jointly by professional astronomers and astronomy teachers and finally recommended by the Ministry of Education for all secondary schools. Alternative programmes also could be implemented through the Ministry of Education if any school justifies its necessity, and several schools with deeper education in the field of physics and mathematics use this opportunity.

However both the secondary and elementary schools suffer from the shortage of astronomy teachers, which is negatively affecting the education quality. Since only an hour per week is allotted for teaching the subject of astronomy, which obviously is not a high enough workload to hire specialized astronomy teachers at every school. Hence this course is taught very often by physicists or other specialists. For a decade in the 1980s, this problem was partially solved using the Yerevan planetarium facilities, with professional lecturers invited from Byurakan Astrophysical Observatory (BAO) and Yerevan State University (YSU). These specialists had elaborated and have been implementing a series of lectures to cover all the main topics of the astronomy course taught in the secondary schools. Unfortunately since the very beginning of the 1990s, the planetarium ceased its operation and there are negligible chances for its reopening in the near future.

Many schools partly compensate this lack of qualified teachers organizing visits to the Byurakan observatory for pupils where they can watch telescopes and also attend short lectures on astronomy, its role and purposes. At present the guide group consists of professional astrophysicists and they receive several thousands of guests per year, included among them nearly 80 per cent of school pupils. Though there is a special gallery in the building of the 2.6-m telescope for receiving visitors, where they attend a lecture and watch the largest BAO telescope, the observatory administration is seeking for a funding to build a new pavilion equipped with a special hall for lectures, as well as two small telescopes for observations etc. In a few schools facultative training is organized by the astronomy amateurs, for the purpose of a deeper learning of astronomy.

At the present date, the most serious danger for astronomical education is related, strange though it may seem, with the process of integration of the country's educational system into the European framework. This trend threatens that astronomy might be 
dropped from the list of mandatory subjects. In such a case, the level of astronomy knowledge would go down very rapidly. The majority of pupils who are presently learning astronomy are more or less doing so owing to the demands of the educational programme and they gain an acceptable knowledge because of that. But only a small part of them will follow their natural intellectual curiosity to learn astronomy after such a decision to make astronomy no longer mandatory. Evidently this process is going on to the detriment of knowledge in astronomy which maybe will slow down but survive only because the Byurakan observatory is well recognized by the population of the country.

\section{Byurakan Astrophysical Observatory - BAO}

$\mathrm{BAO}$ - the main center for astrophysical researches in Armenia was established in 1946 on the initiative of Professor Victor Ambartsumian (IAU President 1961-64; ICSU President 1968-72) who became the first director of the observatory and was occupying this position up to 1988. The main research directions were determined by him as well. The first studies at the Byurakan Astrophysical Observatory were related with the instability phenomena taking place in the universe and their role in the evolution of cosmic objects. This trend became the main characteristic of the research activity in Byurakan and almost all the well-known results obtained at BAO are related to this scientific direction.

First scientific results were announced in 1947 owing to the studies of a new type of stellar systems - stellar associations. Using the dynamical features stellar associations it was shown that these systems were not older than tens of millions of years, proving thus that star-forming processes are going on at present in our Galaxy. Proving the existence of these young stars, Ambartsumian actually made the universe a living organism for the first time. Though this idea met with undisguised skepticism by the astronomical community then, but not any astronomer at present has doubts concerning the existence of young or newborn stars in our Galaxy and other galaxies.

Another very fruitful idea put forward in the Byurakan observatory concerning the activity in galactic nuclei was rejected by the world astronomical community in the beginning of 1960s. However later on, when many new active galaxies have been revealed and, particularly, the Markarian survey was implemented and his catalogue of UV-excess galaxies was completed, a new very wide and rapidly developing scientific direction in extragalactic astronomy was established called AGN studies.

Undoubtedly the fact of existence of the rather famous professional observatory in the country with its scientific achievements, with modern scientific facilities including the 1-m Schmidt telescope and another 2.6-m telescope, being unique ones in the region, and a series of smaller telescopes has had a very strong influence on the educational process. However, it should be mentioned that for a long time there existed an essential gap between professional astronomy and the general educational level in astronomy. Until recently, no amateur astronomy existed in the country in any sense. Now the situation is slightly different, although it needs a long way of improvement still.

For decades, competitions on knowledge of various subjects have been organized for pupils in Armenia, and such competitions made them study these subjects more intensively. Unfortunately astronomy has not been included. Now this problem is solved. During recent years, annual competitions for revealing gifted pupils in astronomy are also organized by the Ministry of Education in collaboration with BAO. These competitions have three rounds, namely, in schools, in districts and the final one as a rule is held at BAO. The country's winners successfully participate and win prestigious prizes in the international astronomical Olympiads as well. In September 2005, an astronomical school-competition was organized at Byurakan where pupils from Russia took part 
as well. The Armenian Ministry of Education with BAO intend to apply for organizing an international Olympiad in Armenia in 2008 - the year when V. Ambartsumian's centenary will be celebrated in Armenia.

\section{Astronomy at Yerevan State University - YSU}

At YSU a department for astrophysics was set up by Prof V. Ambartsumian in 1945 and is operating to date. This department trains specialists for a career in astrophysics. Only one or two students graduate from this department yearly at present, while in the 1980s a dozen students were trained every year. Actually this department was established for a very definite purpose - to train the scientific staff for the future Armenian astrophysical observatory. The observatory was set up in the following year and BAO since then and up to now serves as a scientific base for the students of YSU as well. Mainly the BAO staff members conduct special courses for the students of the mentioned department.

YSU provides a master's degree in astrophysics, and BAO is granting a doctor's (PhD) degree since the $1970 \mathrm{~s}$. It is worth noting that many post-graduate students from various countries of the former Soviet Union,, as well as from abroad, received their $\mathrm{PhD}$ degrees in astrophysics at the Byurakan Astrophysical Observatory. The teachers working at present in the secondary schools of the republic are graduated mainly from the Armenian State Pedagogical University (ASPU) and a smaller part of them are from YSU. The BAO makes plans for organizing with the Ministry of Education at least short term regular courses for the astronomy teachers aimed at increasing their professional skills.

One of the main goals of the recently organized Armenian Astronomical Society (ArAS, 2001) is supporting astronomical education, as well as the propagation of astronomical knowledge within the population in the country. The Society intends to use public lectures as well as opportunities given by TV channels and newspapers for achieving their goals in this particular field.

In 2004 BAO renewed summer training for the students from YSU at the observatory, which was interrupted in the mid-1990s. In 2005 was organized a summer school for the best students of YSU physical faculty, which was aimed at helping them for further choosing of their specialization. Lectures on various subjects were organized for them and also they participated in a competition to show their knowledge. BAO and YSU agreed to organize such schools annually. This year it will be combined with the First Byurakan International School already announced for young astronomers and to be held in the period 22-31 August 2006. 